\title{
LncRNA FAM83H-AS1 promotes aerobic glycolysis and tumor progression by regulating miR-4684- 5p/ZBTB38 axis in esophageal squamous cell carcinoma
}

\section{Cuijuan Qian}

Taizhou University

\section{Zhurong Xu}

Taizhou University

\section{Luyan Chen}

Taizhou University

\section{Yichao Wang}

Taizhou University Hospital

Jun Yao ( $\sim$ yaojuntzu@yeah.net)

Taizhou University https://orcid.org/0000-0002-1088-1548

\section{Primary research}

Keywords: ESCC, glycolysis, FAM83H-AS1, ZBTB38, miR-4684-5p

Posted Date: October 7th, 2020

DOI: https://doi.org/10.21203/rs.3.rs-31503/v2

License: (c) (i) This work is licensed under a Creative Commons Attribution 4.0 International License.

Read Full License 


\section{Abstract}

Background: Dysregulation of IncRNAs is implicated in esophageal squamous cell carcinoma (ESCC) progression; However, the precise function of IncRNA FAM83H-AS1 in ESCC remains unknown. Methods: FAM83H-AS1, miR-4684-5p and ZBTB38 mRNA expressions were detected via qRT-PCR. ZBTB38, GLUT1 and LDH-A protein expressions were tested via Western blot. Cell proliferation, migration and invasion were evaluated via CCK-8 and transwell assay, respectively. A nude mouse xenograft model was used to investigate the role of FAM83H-AS1 in xenograft ESCC growth. The metabolic shift in ESCC cells was examined via glycolysis analysis. The interaction between FAM83H-AS1, miR-4684-5p and ZBTB38 was analyzed via computational algorithms, RNA pull-down, RIP and dual luciferase reporter assay. Results: We found that FAM83H-AS1 was upmodulated in ESCC cell lines. FAM83H-AS1 knockdown hampered ESCC cell proliferation, migration, invasion and aerobic glycolysis, while FAM83H-AS1 overexpression demonstrated the opposite effects. FAM83H-AS1 knockdown also delayed the tumor growth in vivo. Moreover, FAM83H-AS1 interacted with miR-4684-5p/ZBTB38 axis in ESCC cells. ZBTB38 overexpression or miR-4684-5p inhibition partially reversed the inhibitory effect of FAM83H-AS1 knockdown on cell migration, invasion and aerobic glycolysis in ESCC cells. Conclusion: Our present results indicate FAM83H-AS1 accelerated aerobic glycolysis and tumorigenesis of ESCC by sponging miR-4684-5p and triggering the expression of ZBTB38, providing new insights into mechanism of ESCC progression and therapeutic strategy.

\section{Background}

Esophageal squamous cell carcinoma (ESCC) accounts for about $90 \%$ of esophageal cancer, with its incidence on the rise [1, 2]. Although the global mortality rates from ESCC have decreased over the past several decades, many ESCC patients have very poor prognosis because they are diagnosed at an advanced stage $[3,4]$. Hence, early diagnosis and treatment are critical for the management of ESCC patients. As a result, it is urgent to explore novel diagnostic biomarkers and therapeutic targets for ESCC.

Recently, increasing findings support that long noncoding RNAs (IncRNAs) are crucial regulators in ESCC formation and progression [5-10]. Some IncRNAs modulate ESCC cell migration and invasion, and might be novel prognostic indicators, specifically, biomarkers, for the early detection of metastasis in ESCC [5, 9]. For instance, IncRNA DLX6 antisense RNA 1 (DLX6-AS1) expression was significantly increased in ESCC tissues, and DLX6-AS1 knockdown inhibited ESCC cell proliferation, migration and invasion [6]. LncRNAs could affect ESCC progression by modulating axes of miRNAs/miRNA-target genes. For instance, IncRNA myocardial infarction associated transcript (MIAT) promoted ESCC progression by miR-1301-3p/INCENP axis [8], and IncRNA prostate cancer associated transcript-1 (PCAT-1) promoted ESCC progression via miR-508-3p/ANXA10 axis [10]. It has been recently reported that increased expression of IncRNA FAM83H antisense RNA 1 (head to head) (FAM83H-AS1) was observed in triplenegative breast cancer (TNBC) tissues and cells, and overexpression of FAM83H-AS1 promoted TNBC cell proliferation, migration and invasion [11]. However, up to data, the function and related mechanism of IncRNA FAM83H-AS1 has not been systematically studied in ESCC. Therefore, we here provided strong 
evidences to support the promoting role of FAM83H-AS1 in aerobic glycolysis and tumor progression of ESCC.

LncRNAs can function as miRNA sponges for the miRNA-dependent gene silencing in ESCC [5].

Bioinformatics analyses by TargetScan and miRDB predicted that FAM83H-AS1 could interact with miR4684-5p, and miR-4684-5p can directly target zinc finger and BTB domain containing 38 (ZBTB38). However, the influence of miR-4684-5p on tumors has not been reported so far. It is noteworthy that as a transcription factor, ZBTB38 is involved in human neuroblastoma cell regulation, proliferation and apoptosis, whereas knockdown of ZBTB38 induces neuroblastoma cell death potentially [12]. ZBTB38 may promote cell migration and invasion of bladder cancer cells via up-modulation of Wnt/ $\beta$-catenin pathway [13]. The above findings suggest that ZBTB38 may function as an oncogene in some tumors. Nevertheless, it is not clear whether FAM83H-AS1 can play a role in ESCC by modulating miR-4684-5p and/or ZBTB38.

The key role of the aerobic glycolysis in tumors was elucidated in the past decade [14, 15]. Enhanced glycolysis is one of the characteristics of ESCC and other tumors as the tumor cells rely on the glycolytic pathway for their energy needs $[16,17]$. This characteristic of cell metabolism has been successfully exploited for cancer diagnosis and therapy [18]. Many proteins, such as lactate dehydrogenase-A (LDH-A) and glucose transporter type 1 (GLUT1), control the irreversible steps in glycolysis catalyze reactions, and are considered as key enzymes of aerobic glycolysis $[19,20]$. However, how IncRNAs modulate glycolysis by interacting with the key enzymes of aerobic glycolysis is still poorly understood.

In the present study, the effects of FAM83H-AS1 on glycolysis in ESCC cell lines and role of FAM83HAS1/miR-4684-5p/ZBTB38 axis was systematically investigated in ESCC. Our study suggests a novel role and mechanism that FAM83H-AS1/miR-4684-5p/ZBTB38 axis contributed to glucose metabolism and tumor progression of ESCC.

\section{Materials And Methods}

\section{Cell lines and cell culture}

The human ESCC cell lines EC9706, EC109, KYSE30 and KYSE150, and a human esophageal squamous epithelial cell line Het-1A were obtained from the Chinese Academy of Sciences Committee on Type Culture Collection Cell Bank (Shanghai, China). The cells were cultured in Roswell Park Memorial Institute 1640 medium (RPMI-1640; Gibco, Carlsbad, CA, USA) supplemented with $10 \%$ fetal bovine serum (FBS; Sigma-Aldrich, St. Louis, $\mathrm{MO}, \mathrm{USA}$ ) at $37^{\circ} \mathrm{C}$ in a $5 \% \mathrm{CO}_{2}$ atmosphere.

\section{RNA isolation and quantitative real-time polymerase chain reaction (qRT-PCR)}


Total cellular RNA was extracted using TRIzol reagent (Invitrogen, Waltham, CA, USA). For miRNA expression analysis, qRT-PCR was carried out using the TaqMan MicroRNA Reverse Transcription kit and TaqMan Universal PCR Master Mix (Applied Biosystems, Foster City, CA, USA). The relative quantification of miR-4684-5p was performed using the $2^{-\triangle \triangle C t}$ method, with U6 used as an internal control. For FAM83H-AS1 and ZBTB38 expression analysis, qRT-PCR was performed using the TaqMan High-Capacity cDNA Reverse Transcription Kit and TaqMan Fast PCR Master Mix (Applied Biosystems, Foster City, CA, USA) according to the manufacturer's instructions. The relative quantification of FAM83H-AS1 and ZBTB38 was performed using the $2^{-\triangle \triangle C t}$ method, with GAPDH used as an internal control. The reactions were performed independently in triplicate, and the primer sequences are listed in Table 1. The RNA expression levels were analyzed as described previously [13, 21].

\begin{tabular}{|ll|}
\hline Name & Primer sequence \\
\hline FAM83H-AS1 & F: 5'-TAGGAAACGAGCGAGCCC-3' \\
& R: 5'-GCTTTGGGTCTCCCCTTCTT-3' \\
'BR-4684-5p & F: 5'-ACCAGGGGTACCTCTCTACT-3' \\
& R: 5'-AAGGGTACGTCTCCACCGA-3' \\
& F: 5'-TGTCTTGAAGTGAGGCTCTGCTG-3' \\
GAPDH & F: 5'-AGCAAGCCTTGTGGACCAAAC-3' \\
& R: 5'-GCCTTCTCCATGGTGGTGAA-3' \\
\hline U6 & F: 5'-GCTTCGGCAGCACATATACTAAAAT-3' \\
& R: 5'-CGCTTCACGAATTTGCGTGTCAT-3' \\
\hline
\end{tabular}

Table 1

qRT-PCR primer sequences

\section{Cell transfection}

FAM83H-AS1 was cloned into empty pcDNA3.1 vectors (Invitrogen, Waltham, MA, USA) to construct FAM83H-AS1 overexpressing plasmid (p-FAM83H-AS1). The empty pcDNA3.1 vector was used as a control (vector control). Small interfering RNAs (siRNAs) targeting FAM83H-AS1 (si-FAM83H-AS1), negative control siRNAs (si-NC), miR-4684-5p inhibitors, miR-4684-5p mimics and miRNA scrambled control (control mimics) were all synthesized by GenePharma (Shanghai, China). All of the above vectors were subsequently transfected into ESCC cells by applying Lipofectamine 2000 (Invitrogen, Waltham, MA, USA) according to the manufacturer's manual. After $48 \mathrm{~h}$ of transfection, cells were collected and processed for further studies. 


\section{Cell proliferation assay}

After transfection, ESCC cell viability was assessed with the CCK-8 kit (Dojindo, Kumamoto, Japan). In brief, ESCC cells $\left(3 \times 10^{3}\right.$ cells $/ 200 \mu \mathrm{l} /$ well $)$ were seeded into the 96 -well plates, and six replicates were set up for each sample. $100 \mu \mathrm{l}$ of fresh medium were added to replace culture medium at the appointed time point, and $10 \mu \mathrm{l}$ of CCK-8 solution were pipetted into each well immediately. After incubated at $37^{\circ} \mathrm{C}$ for $2 \mathrm{~h}$, the OD450 value were determined using a microplate reader (Bio-Rad Laboratories, Inc., Hercules, CA, USA).

\section{Cell migration and invasion assay}

ESCC cell migration was evaluated using a transwell migration assay. $5 \times 10^{4}$ ESCC cells/well were resuspended in $250 \mu \mathrm{l}$ RPMI 1640 supplemented with 1\% FBS and plated onto uncoated 8- $\mu \mathrm{m}$ transwell filter inserts in 24-well plates in triplicate. The lower chambers contained $500 \mu \mathrm{l}$ of RPMI 1640 supplemented with $15 \%$ FBS as a chemoattractant. The non-migratory cells in the upper chamber were removed with a cotton swab after incubation for $16 \mathrm{~h}$, while cells on the bottom side were fixed in $100 \%$ methanol and stained with $0.5 \mu \mathrm{g} / \mathrm{ml}$ 4',6-diamidino-2-phenylindole (DAPI) for $5 \mathrm{~min}$. Then stained cells were counted using a fluorescence microscope (Eclipse 80i; Nikon Corporation, Tokyo, Japan) in five random fields.

A transwell invasion assay was performed to assess cell invasion ability. ESCC cells were seeded into the upper chamber of Matrigel-coated inserts containing $250 \mu$ l RPMI 1640 containing 1\% FBS. RPMI 1640 containing $15 \%$ FBS was added to the lower chamber as a chemoattractant. After incubation for $48 \mathrm{~h}$, cells in the upper chamber were removed, and cells on the bottom side of chamber were fixed in $70 \%$ ethanol and stained with $0.1 \%$ crystal violet for $30 \mathrm{~min}$. The stained cells were lysed with $200 \mu \mathrm{l}$ of lysis reagent. Finally, $100 \mu \mathrm{l}$ of lysate was taken to a 96 -well plate, and the absorbance was measured at 560 $\mathrm{nm}$ by a microplate reader (550; Bio-Rad, USA).

\section{Glucose uptake and lactate production assay}

ESCC cells were cultured in glucose-free RPMI 1640 for $16 \mathrm{~h}$, and then incubated with high-glucose RPMI 1640 under normoxic conditions for an additional $24 \mathrm{~h}$. Culture supernatants were then collected for intracellular glucose levels measure using a fluorescence-based glucose assay kit (BioVision, Milpitas, California, USA) according to the manufacturer's instructions. Lactate levels were measured using a lactate oxidase-based colorimetric assay read at $540 \mathrm{~nm}$ according to the manufacturer's instructions (Beyotime, Wuxi, China) and normalized to cell number.

\section{Bioinformatics analysis}


The potential target miRNAs of FAM83H-AS1 were predicted via computational algorithms, including TargetScan (http://www.targetscan.org/vert_72/) and miRDB (http://mirdb.org/). The highest-ranked predicted potential target of FAM83H-AS1 was miR-4684-5p. To identify potential genes targeted by miR-4684-5p, we also used the TargetScan and miRDB. From the list of target genes obtained, all genes likely to contribute to ESCC progression were extracted.

\section{Luciferase reporter assay}

The partial sequences of FAM83H-AS1 or ZBTB38 3'-UTR, which contains the putative miR-4684-5pbinding site, were amplified by PCR and constructed into the pmirGLO Luciferase vector (Promega, Madison, WI, USA) to generate wild-type FAM83H-AS1 reporter (FAM83H-AS1-WT) or ZBTB38 reporter (ZBTB38-WT). The GeneArt ${ }^{\text {TM }}$ Site-Directed Mutagenesis System (Thermo Fisher Scientific, Waltham, MA, USA) was used to produce mutant-type FAM83H-AS1 (miR-4684-5p target site-mutation FAM83H-AS1, FAM83H-AS1-MUT) reporter or mutant-type ZBTB38 (miR-4684-5p target site-mutation ZBTB38 3'-UTR, ZBTB38-MUT) reporter. All constructs were verified by DNA sequencing. Subsequently, the luciferase reporter and control mimic or miR-4684-5p mimic were co-transfected into ESCC cells. $48 \mathrm{~h}$ after transfection, luciferase assays were performed using the Dual Luciferase Reporter Assay System (Promega, Madison, WI, USA) following the manufacturer's instructions.

\section{RIP assay}

RIP was performed using a Magna RIP RNA-Binding Protein Immunoprecipitation Kit (Millipore, MA, USA) following the manufacturer's instructions. In brief, $1 \times 10^{7}$ ESCC cells transfected with miR-4684-5p mimic and control mimic were lysed in the RIP lysis buffer containing a protease inhibitor cocktail. Next, the ESCC cell supernatant was incubated with the RIP buffer containing a magnetic bead conjugated with antibodies against human AGO2 or the control normal mouse IgG. Then the protein and DNA in the RIP complex were removed using RNase-free DNase I and Proteinase $\mathrm{K}$. Then, the immunoprecipitated RNA complex was isolated and subjected to qRT-PCR to detect the enrichment of FAM83H-AS1 or ZBTB38.

\section{RNA pull-down assay}

ESCC cells were transfected with biotin-labeled wild type miR-4684-5p (Bio-miR-4684-5p-WT), mutated miR-4684-5p (Bio-miR-4684-5p-MUT) or antagonistic miR-4684-5p probe (GenePharma, Shanghai, China). $48 \mathrm{~h}$ after transfection, cells were harvested and incubated with a specific lysate buffer (Ambion, Austin, TX, USA) for $10 \mathrm{~min}$. Then, the cell lysates were mixed with $\mathrm{M}-280$ streptavidin magnetic beads (Invitrogen, Waltham, CA, USA) for $3 \mathrm{~h}$ at $4{ }^{\circ} \mathrm{C}$. The pull-down products were subjected to qRT-PCR for FAM83H-AS1 expression. 


\section{In vivo xenograft experiments}

The animal experiments were approved by the Animal Care and Use Committee of Taizhou University School of Medicine and performed in accordance with the relevant guidelines and regulations of the committee. BALB/c athymic nude mice (female, 4 weeks old) were purchased from Shanghai Laboratory Animal Center (Shanghai, China) and randomly divided into three groups (Blank control, si-FAM83H-AS1 and si-NC; $\mathrm{n}=5$ each). A total of $1 \times 10^{7}$ EC9706 cells (trancfected with si-FAM83H-AS1 or si-NC, untreated cells as blank control, respectively) were subcutaneously injected into one flank of each nude mouse. Tumor volume was calculated using the formula (Length $\times$ Width $\left.{ }^{2}\right) / 2$. Tumors were harvested and weighed after $24 \mathrm{~d}$.

\section{Western blotting analysis}

Cells were collected and lysed using RIPA Lysis Buffer (Beyotime, Wuxi, China) supplemented with a protease inhibitor cocktail and phenylmethylsulfonyl fluoride (Roche, Pleasanton, CA, USA). The protein concentration was determined using a BCA Protein Assay Kit (Beyotime, Wuxi, China). Approximately 50 $\mu \mathrm{g}$ of protein extract was separated on $10 \%$ sodium dodecyl sulfate-polyacrylamide gel electrophoresis (SDS-PAGE) gels, transferred to polyvinylidene fluoride (PVDF) membranes (Merck KGaA, Darmstadt, Germany) and incubated with specific antibodies. The primary antibodies against ZBTB38 (1:1000, catalog no.ab231263; Abcam, Cambridge, MA, USA), LDH-A (1:1000, catalog no.ab125683; Abcam, Cambridge, MA, USA) and GLUT1 (1:1000, catalog no.ab15309; Abcam, Cambridge, MA, USA) were used. Following extensive washing, membranes were incubated with a horseradish peroxidase-conjugated goat polyclonal anti-rabbit IgG secondary antibody (1:2000, catalog no.7074; Cell Signaling Technology, Danvers, MA, USA) for $1 \mathrm{~h}$ at room temperature. Immunoreactivity was detected by enhanced an chemiluminescence system kit (Pierce; Thermo Fisher Scientific, Inc., Waltham, MA, USA) and visualized using an LAS-4000 imaging system (Fujifilm Holdings Corporation, Tokyo, Japan). GAPDH (1:1000, catalog no.ab181602; Abcam, Cambridge, MA, USA) served as a loading control.

\section{Statistical analysis}

All data are presented as the mean \pm standard deviation (SD). The statistical analyses were performed using SPSS 18.0 software (IBM, New York, USA). Differences between groups were analyzed using Student's t-test (two groups) or one-way ANOVA (multiple groups). $\mathrm{P}<0.05$ was considered statistically significant.

\section{Results}

\section{FAM83H-AS1 was upmodulated and facilitated cell proliferation in ESCC cells}


qRT-PCR analysis showed that FAM83H-AS1 expression was upmodulated in four ESCC cell lines compared with that in Het-1A (Fig.1a). Then, FAM83H-AS1 was knocked down by FAM83H-AS1 siRNA transfection in EC9706 cells (Fig.2b), and overexpressed by p-FAM83H-AS1 transfection in EC109 cells (Fig.2c). CCK-8 assay showed that FAM83H-AS1 knockdown repressed the EC9706 cell proliferation (Fig.2e), while FAM83H-AS1 overexpression promoted EC109 cell proliferation (Fig.2f). In vivo heterotransplantation mice assay illustrated that FAM83H-AS1 knockdown repressed the tumor growth of EC9706 cells (Fig.2f,g). Overall, the above findings suggested that FAM83H-AS1 positively modulates the cell proliferation of ESCC cells in vitro and in vivo.

\section{FAM83H-AS1 facilitated cell invasion and migration in ESCC cells}

Moreover, transwell assay with or without Matrigel demonstrated that FAM83H-AS1 knockdown significantly restrained the cell invasion (Fig.2a) and migration (Fig.2b,c) in EC9706 cells, while FAM83HAS1 overexpression significantly facilitated the cell invasion (Fig.2d) and migration (Fig.2e,f) in EC109 cells. Overall, these findings indicated that FAM83H-AS1 positively modulates the cell invasion and migration in ESCC cells.

\section{FAM83H-AS1 facilitated the aerobic glycolysis in ESCC cells}

To explore whether FAM83H-AS1 influences the malignant phenotype of ESCC cells by modulating the aerobic glycolysis, the effects of FAM83H-AS1 on glucose uptake and lactate production were identified by glycolysis analysis. As shown in Fig.3a-d, knockdown of FAM83H-AS1 significantly suppressed the glucose uptake (Fig.3a) and lactate production (Fig.3b) in EC9706 cells, while overexpression of FAM83HAS1 showed the opposite effects in EC109 cells (Fig.3c,d).

qRT-PCR and Western blotting analysis were performed to detect the expression of aerobic glycolysis associated proteins in ESCC cells. The results revealed that FAM83H-AS1 knockdown decreased GLUT1 and LDH-A mRNA and protein expression in EC9706 cells (Fig.3e-g). In contrast, FAM83H-AS1 overexpression led to increased GLUT1 and LDH-A mRNA and protein expression in EC109 cells (Fig.3h-j). These above results indicated that FAM83H-AS1 can induce the aerobic glycolysis of ESCC cells by positively modulating GLUT1 and LDH-A expression.

\section{FAM83H-AS1 acts as a sponge of miR-4684-5p in ESCC cells}


Bioinformatics analysis indicated that the top four predicted potential target miRNAs of FAM83H-AS1 are $m i R-4684-5 p, m i R-10397-3 p, m i R-5196-3 p$ and miR-4478 (Table 2). The results of qRT-PCR showed that FAM83H-AS1 knockdown in EC9706 cells accelerated the expressions of miR-4684-5p, miR10397-3p, miR-5196-3p and miR-4478, respectively, among which miR-4684-5p was the most obvious (Fig.4a). The binding sites that miR-4684-5p might shared with FAM83H-AS1 were showed in Fig.4b.

\begin{tabular}{|lll|}
\hline Target Rank & Target Score & miRNA Name \\
\hline 1 & 96 & hsa-miR-4684-5p \\
\hline 2 & 92 & hsa-miR-10397-3p \\
\hline 3 & 90 & hsa-miR-5196-3p \\
\hline 4 & 88 & hsa-miR-4478 \\
\hline 5 & 87 & hsa-miR-520b-5p \\
\hline 7 & 87 & hsa-miR-519a-2-5p \\
\hline 8 & 86 & hsa-miR-6780a-5p \\
\hline 9 & 86 & hsa-miR-1283 \\
\hline 10 & 86 & hsa-miR-5694 \\
\hline 11 & 85 & hsa-miR-6809-5p \\
\hline
\end{tabular}

Table 2

The predicted targets of FAM83H-AS1

Subsequently, the double luciferase reporter assay demonstrated that co-transfection of miR-4684-5p mimics and pmirGLO-FAM83H-AS1-WT vector (without mutation at the target site) could significantly reduce luciferase activity in EC9706 cells (Fig.4c), while co-transfection of miR-4684-5p mimics and pmirGLO-FAM83H-AS1-MUT vector (with mutations in the targeting site) did not affect luciferase activity, indicating that miR-4684-5p could bind with FAM83H-AS1 at the molecular level in EC9706 cells. To further validate the interaction between miR-4684-5p and FAM83H-AS1, RNA pull-down assay was performed using biotinylated miR-4684-5p-WT and miR-4684-5p-MUT probes. As shown in Fig.4d, only miR-4684-5p-WT could bind and precipitate FAM83H-AS1 in cell lysates of EC9706 cells, but there was no interaction between FAM83H-AS1 and miR-4684-5p-MUT or biotin-labeled antagonistic miR4684-5p probe (Bio-NC). To determine whether FAM83H-AS1 binds miR-4684-5p in an AG02dependent manner, we performed anti-AG02 RIP in EC9706 cells overexpressing miR-4684-5p. FAM83HAS1 enrichment was increased after overexpression of miR-4684-5p in EC9706 cells (Fig.4e). Taken together, the above results suggested that FAM83H-AS1 may sponge miR-4684-5p and function as a ceRNA in ESCC. 


\section{miR-4684-5p directly targeted ZBTB38 in ESCC cells}

Further, the two online database, TargetScan and miRDB, were also used to predict potential target genes of miR-4684-5p. From the list of target genes obtained, all genes likely to contribute to ESCC progression were extracted (Table 3). We therefore overexpressed these above four miRNAs (miR-4684$5 p$, miR-10397-3p, miR-5196-3p and miR-4478) in EC9706 cells by transfecting miRNA mimics. Interestingly, only miR-4684-5p overexpression significantly suppressed mRNA expression of ZBTB38 in EC9706 cells (Fig.5a).

\begin{tabular}{|llll|}
\hline Target Rank & Target Score & Gene Symbol & Gene Description \\
\hline 1 & 98 & JADE2 & jade family PHD finger 2 \\
\hline 2 & 98 & GALNT15 & polypeptide N-acetylgalactosaminyltransferase 15 \\
3 & 98 & ZBTB38 & zinc finger and BTB domain containing 38 \\
\hline 4 & 96 & SPATA31A6 & SPATA31 subfamily A member 6 \\
\hline 6 & 96 & CXXC5 & CXXC finger protein 5 \\
\hline 7 & 96 & AMTN & amelotin \\
\hline 9 & 96 & SPATA31A7 & SPATA31 subfamily A member 7 \\
\hline 10 & 96 & SPATA31A3 & SPATA31 subfamily A member 3 \\
\hline
\end{tabular}

Table 3

The predicted targets for miR-4684-5p

Based on above bioinformatics analyses and relevant references, the 3'-UTR of ZBTB38 was predicted to have miR-4684-5p-binding sites, suggesting that ZBTB38 had the higher associability with miR-4684$5 p$ (Fig.5b). Furthermore, RIP assay revealed a higher level of ZBTB38 enrichment in the AGO2 group after overexpression of miR-4684-5p in EC9706 cells (Fig.5c). Luciferase reporter assay confirmed that miR4684-5p covalently targeted ZBTB38 mRNA 3'-UTR in EC9706 cells (Fig.5d). Thus, we here found that miR-4684-5p has a direct effect on ZBTB38.

\section{FAM83H-AS1 negatively modulated miR-4684-5p expression and positively modulated ZBTB38 expression in ESCC cells}

qRT-PCR assay indicated that knockdown of FAM83H-AS1 increased miR-4684-5p expression (Fig.6a) and decreased ZBTB38 mRNA expression (Fig.6b) in EC9706 cells, while overexpression of FAM83H-AS1 
decreased miR-4684-5p expression (Fig.6c) and increased ZBTB38 mRNA expression (Fig.6d) in EC109 cells. Western blot analysis showed that knockdown of FAM83H-AS1 decreased ZBTB38 expression in EC9706 cells (Fig.3g), while overexpression of FAM83H-AS1 increased ZBTB38 expression in EC109 cells (Fig.3j). Further qRT-PCR assay indicated that inhibition of miR-4684-5p increased ZBTB38 mRNA expression in EC109 cells (Fig.6e), while overexpression of miR-4684-5p decreased ZBTB38 mRNA expression in EC9706 cells (Fig.6f). Taken together, our results indicated that FAM83H-AS1 negatively modulated miR-4684-5p expression and positively modulated ZBTB38 expression, and miR-4684-5p negatively modulated ZBTB38 expression, suggesting FAM83H-AS1 may facilitate ZBTB38 expression by down-modulating miR-4684-5p.

\section{miR-4684-5p inhibition or ZBTB38 overexpression partially reverses the inhibitory effects of FAM83H-AS1 knockdown on ESCC cells}

To explore the role of miR-4684-5p and ZBTB38 in FAM83H-AS1 modulating cell migration, invasion and glycolysis, rescue assays were further carried out in EC9706 cells. The cell invasion (Fig.7a) and migration (Fig.7b) were significantly hampered by FAM83H-AS1 knockdown, and then it was partially reversed after transfection with miR-4684-5p inhibitor or pZBTB38 (Fig.7a,b). Meanwhile, the glucose uptake (Fig.7c) and lactate production (Fig.7d) were significantly hampered by FAM83H-AS1 knockdown, and then it was partially reversed after transfection with miR-4684-5p inhibitor or pZBTB38 (Fig.7c,d). Thus, the above results showed that miR-4684-5p inhibition or ZBTB38 overexpression could partially reverse the inhibitory effects of FAM83H-AS1 knockdown on cell migration, invasion and glycolysis in ESCC cells, suggesting FAM83H-AS1 may modulate the malignant behaviors of ESCC cells via miR4684-5p/ZBTB38 axis.

\section{Discussion}

The role of IncRNAs in ESCC formation and progression has gradually emerged [5]. LncRNA FAM83H-AS1 has been reported to play an oncogenic role in tumors by modulating cell proliferation, migration and invasion [11, 21-24]. However, the role and mechanism of FAM83H-AS1 contributing to ESCC formation and progression has not been elucidated. In the present study, we first found that FAM83H-AS1 expression was significantly higher than that of human esophageal squamous epithelial cell line Het-1A (Fig.1a), indicating that FAM83H-AS1 might contribute to the malignant nature of ESCC cells. The present mechanism experiments revealed the interaction between FAM83H-AS1, miR-4684-5p and ZBTB38 in ESCC progression (Fig.4,5). Then gain- and loss-of-function (Fig.1-3) and rescue experiments (Fig.7) verified a promoting effect of FAM83H-AS1 on ESCC cell migration, invasion and aerobic glycolysis by modulating miR-4684-5p/ZBTB38 axis .

Previous studies suggest that aerobic glycolysis is implicated in the malignant behaviors of ESCC [2527]. Enhanced aerobic glycolysis is a striking feature of ESCC which relays upon the glycolytic pathway 
for their energy needs [25-27]. Moreover, increasing data have revealed that IncRNAs modulate tumor invasion and metastasis by affecting the aerobic glycolysis [28]. Thus, as important and extensive regulators of the aerobic glycolysis, IncRNAs might be suitable candidates for ESCC diagnosis and treatment. Here, we found that overexpression of FAM83H-AS1 not only accelerated the cell migration and invasion of ESCC cells (Fig.2), but also facilitated glucose uptake and lactate production (Fig.3), suggesting that the acquisition of metastastic potential of ESCC induced by FAM83H-AS1 might benefit from glucose metabolism. Therefore, we hypothesized that FAM83H-AS1 may influence the malignant phenotype of ESCC partly by modulating the aerobic glycolysis. A number of enzymes are involved in the aerobic glycolysis process, including GLUT1 and LDH-A $[19,29]$. LDH-A can accelerate the invasion and proliferation of pituitary adenoma through the upmodulation of GLUT1 [30]. Thus, we determined the GLUT1 and LDH-A expressions following overexpression or downmodulation of FAM83H-AS1, and found that knockdown or overexpression of FAM83H-AS1 decreased or increased the expressions of GLUT1 and LDH-A (Fig.3), as well as the downmodulation or upmodulation of glucose uptake and lactate production levels, indicating that FAM83H-AS1 may affect aerobic glycolysis process of ESCC partly by modulating GLUT1 and LDH-A.

The competing endogenous RNAs (ceRNAs) hypothesis proposes a novel regulatory network involving IncRNAs, miRNAs, circular RNAs (circRNAs) and pseudogenes, and such a network is of great

significance in tumor formation and progression [31]. Accumulating evidence demonstrated that IncRNAs exerted biological roles in various tumors by functioning as ceRNAs for sponging miRNA to modulating miRNAs' target gene expression [32]. LncRNAs can block the repression of miRNA on its target gene by competitively binding to the miRNA, thus serving as tumor promoters or tumor suppressors [33]. Thus, we supposed that FAM83H-AS1 may exert an oncogenic role in ESCC by functioning as a ceRNA for sponging miRNAs. By use of informatics analysis tools, we screened a few miRNAs sharing binding sites with IncRNA FAM83H-AS1, and found the highest-ranked predicted potential target of FAM83H-AS1 was miR-4684-5p (Table 2, Fig.4a,b). Through luciferase activity, RNA pull-down and RIP assays, the binding relationship between FAM83H-AS1 and miR-4684-5p was identified in ESCC cells (Fig.4c-e). Therefore, miR-4684-5p was chosen for our object, and we speculated that FAM83H-AS1 might serve as a ceRNA to absorb miR-4684-5p.

Since the function of miRNAs are realized by modulating their target genes' mRNA expression [34], we here conducted the bioinformatics analysis and verification experiments (Table 3, Fig.5), and showed that ZBTB38 was the potential downstream target of miR-4684-5p. ZBTB38 is a transcriptional activator that belongs to the zinc finger protein family and contains the typical BTB (for Broad-Complex, Tramtrack and Bric a brac) domains [12]. As a transcription factor, ZBTB38 is involved in cell regulation, proliferation and apoptosis, whereas functional deficiency of ZBTB38 induces the human neuroblastoma cell death [12]. Moreover, ZBTB38 play an oncogenic role in neuroblastoma and bladder cancer [12, 13]. Based on these studies, we hypothesized that FAM83H-AS1 may be partially required for ZBTB38 to exert its oncogenic effect in ESCC. To address this point, the present rescue experiment showed that restoration of ZBTB38 partly blocked FAM83H-AS1 knockdown-induced suppression of cell migration, invasion and 
glycolysis in ESCC cells (Fig.7). Hence, the results suggested that FAM83H-AS1 may promote cell migration, invasion and glycolysis of ESCC cells by modulating ZBTB38.

Increasing studies show that IncRNAs can directly interacts with miRNAs to modulate their downstream target expressions in ESCC $[7,10]$. Here, our bioinformatics analyses predicated potential binding sites in miR-4684-5p and ZBTB38 3'-UTR, as well as FAM83H-AS1 and miR-4684-5p (Fig.4,5). Importantly, our present mechanistic experiments confirmed the binding sites between ZBTB38 3'-UTR and miR-4684-5p (Fig.5), as well as FAM83H-AS1 and miR-4684-5p (Fig.4). Further gain- and loss-of-function and rescue experiments showed that inhibition of miR-4684-5p or restoration of ZBTB38 expression abrogated FAM83H-AS1 knockdown-attenuated the cell migration, invasion and glycolysis of ESCC cells (Fig.6,7). Our present results supported the notion that FAM83H-AS1 acts as a molecular sponge for miR-4684-5p to modulate the expression level of ZBTB38. Therefore, the above findings suggested the possibility that FAM83H-AS1/miR-4684-5p/ZBTB38 may be a novel mechanism responsible for ESCC progression.

\section{Conclusion}

We highlight that FAM83H-AS1 acts as a ceRNA by sponging miR-4684-5p to promote ZBTB38 expression, thereby facilitating the aerobic glycolysis, tumor invasion and metastasis of ESCC (Fig.8), providing new insights into mechanism of ESCC progression and therapeutic strategy. Importantly, we have here revealed a role of FAM83H-AS1 in the aerobic glycolysis through modulation of miR-46845p/ZBTB38 axis. However, the underlying mechanisms that control a shift in ESCC cell metabolism are complex and only partially understood, and the specific mechanisms through which FAM83H-AS1 modulates the aerobic glycolysis require further investigation.

\section{Abbreviations}

FAM83H-AS1: FAM83H antisense RNA 1 (head to head)

ESCC: esophageal squamous cell carcinoma

IncRNA: long non-coding RNA

RIP: RNA binding protein immunoprecipitation

CCK-8: cell counting kit-8

GLUT1: Glucose transporter type 1

LDH-A: lactate dehydrogenase-A

si-NC: negative control siRNAs

qRT-PCR: quantitative real-time PCR 
UTR: untranslated region

ceRNA: competing endogenous RNA

ZBTB38: zinc finger and BTB domain containing 38

\section{Declaration}

\section{Acknowledgements}

None.

\section{Authors' contributions}

Jun Yao and Cuijuan Qian conceptualized and designed the experiments; Cuijuan Qian and Zhurong Xu performed the experiments; Cuijuan Qian, Zhurong Xu, Luyan Chen and Yichao Wang contributed reagents/materials/analysis tools; Cuijuan Qian and Zhurong Xu analyzed the data and wrote the paper; Jun Yao and Cuijuan Qian revised the paper. All authors read and approved the final manuscript.

\section{Funding}

This work was fnancially supported by grants from the Zhejiang Provincial Natural Science Foundation (LY16h260033), the National Natural Science Foundation of China (81902138), the Medical Science and Technology Project of Zhejiang Province (2019RC314), the Science and Technology Plans of Taizhou City (1802ky46) and the National College Students Innovation and Entrepreneurship Training Program (201910350008).

\section{Availability of data and materials}

Please contact authors for data request.

\section{Ethics approval and consent to participate}

The animal experiments were approved by the Committee on Ethics of Animal Experiments of Taizhou University Medical School.

\section{Consent for publication}

All authors approved publication of the manuscript. 


\section{Competing interests}

The authors declare that there are no conflicts of interest.

\section{References}

1. Smyth EC, Lagergren J, Fitzgerald RC, Lordick F, Shah MA, Lagergren P, Cunningham D. Oesophageal cancer. Nat Rev Dis Primers. 2017;3:17048.

2. Abnet CC, Arnold M, Wei WQ. Epidemiology of Esophageal Squamous Cell Carcinoma. Gastroenterology. 2018;154(2):360-373.

3. Lam AK. Introduction: Esophageal Squamous Cell Carcinoma-Current Status and Future Advances. Methods Mol Biol. 2020;2129:1-6.

4. Batra R, Malhotra GK, Singh S, Are C. Managing Squamous Cell Esophageal Cancer. Surg Clin North Am. 2019;99(3):529-541.

5. Feng Q, Zhang H, Yao D, Chen WD, Wang YD. Emerging Role of Non-Coding RNAs in Esophageal Squamous Cell Carcinoma. Int J Mol Sci. 2019;21(1):258.

6. Wu SB, Wang HQ. Upregulation of long noncoding RNA DLX6-AS1 promotes cell growth and metastasis in esophageal squamous cell carcinoma via targeting miR-577. Eur Rev Med Pharmacol Sci. 2020;24(3):1195-1201.

7. Liang M, Pan Z, Yu F, Chen C. Long noncoding RNA SNHG12 suppresses esophageal squamous cell carcinoma progression through competing endogenous RNA networks. Clin Transl Oncol. 2020 Feb 21:10.1007/s12094-020-02317-7. doi: 10.1007/s12094-020-02317-7. Epub ahead of print.

8. Zhang C, Xie L, Fu Y, Yang J, Cui Y. IncRNA MIAT promotes esophageal squamous cell carcinoma progression by regulating miR-1301-3p/INCENP axis and interacting with SOX2. J Cell Physiol. 2020 Jan 14:10.1002/jcp.29448. doi: 10.1002/jcp.29448. Epub ahead of print.

9. Talebi A, Masoodi M, Mirzaei A, Mehrad-Majd H, Azizpour M, Akbari A. Biological and clinical relevance of metastasis-associated long noncoding RNAs in esophageal squamous cell carcinoma: A systematic review. J Cell Physiol. 2020;235(2):848-868.

10. Zang B, Zhao J, Chen C. LncRNA PCAT-1 Promoted ESCC Progression via Regulating ANXA10 Expression by Sponging miR-508-3p. Cancer Manag Res. 2019;11:10841-10849.

11. Han C, Fu Y, Zeng N, Yin J, Li Q. LncRNA FAM83H-AS1 promotes triple-negative breast cancer progression by regulating the miR-136-5p/metadherin axis. Aging (Albany NY). 2020;12(4):35943616.

12. Chen J, Xing C, Yan L, Wang Y, Wang H, Zhang Z, Yu D, Li J, Li H, Li J, Cai Y. Transcriptome profiling reveals the role of ZBTB38 knock-down in human neuroblastoma. PeerJ. 2019;7:e6352.

13. Jing J, Liu J, Wang Y, Zhang M, Yang L, Shi F, Liu P, She J. The role of ZBTB38 in promoting migration and invasive growth of bladder cancer cells. Oncol Rep. 2019;41(3):1980-1990. 
14. Vanhove K, Graulus GJ, Mesotten L, Thomeer M, Derveaux E, Noben JP, Guedens W, Adriaensens P. The Metabolic Landscape of Lung Cancer: New Insights in a Disturbed Glucose Metabolism. Front Oncol. 2019;9:1215.

15. Zhang L, Fu Y, Guo H. c-Myc-Induced Long Non-Coding RNA Small Nucleolar RNA Host Gene 7 Regulates Glycolysis in Breast Cancer. J Breast Cancer. 2019;22(4):533-547.

16. Vaupel $P$, Schmidberger $H$, Mayer A. The Warburg effect: essential part of metabolic reprogramming and central contributor to cancer progression. Int J Radiat Biol. 2019;95(7):912-919.

17. Kobliakov VA. The Mechanisms of Regulation of Aerobic Glycolysis (Warburg Effect) by Oncoproteins in Carcinogenesis. Biochemistry (Mosc). 2019;84(10):1117-1128.

18. Weiss JM. The promise and peril of targeting cell metabolism for cancer therapy. Cancer Immunol Immunother. 2020;69(2):255-261.

19. Koch A, Ebert EV, Seitz T, Dietrich P, Berneburg M, Bosserhoff A, Hellerbrand C. Characterization of glycolysis-related gene expression in malignant melanoma. Pathol Res Pract. 2020;216(1):152752.

20. Hao LS, Liu Q, Tian C, Zhang DX, Wang B, Zhou DX, Li ZP, Yuan ZX. Correlation and expression analysis of hypoxia-inducible factor $1 \mathrm{a}$, glucose transporter 1 and lactate dehydrogenase 5 in human gastric cancer. Oncol Lett. 2019;18(2):1431-1441.

21. Wei R, Chen Y, Zhao Z, Gu Q, Wu J. LncRNA FAM83H-AS1 induces nucleus pulposus cell growth via targeting the Notch signaling pathway. J Cell Physiol. 2019;234(12):22163-22171.

22. Yang L, Cui J, Wang Y, Tan J. FAM83H-AS1 is upregulated and predicts poor prognosis in colon cancer. Biomed Pharmacother. 2019;118:109342.

23. Da J, Liu P, Wang R, Bu L. Upregulation of the long non-coding RNA FAM83H-AS1 in gastric cancer and its clinical significance. Pathol Res Pract. 2019;215(10):152616.

24. Ma YK, Shen TH, Yang XY. Upregulation of LncRNA FAM83H-AS1 in hepatocellular carcinoma promotes cell proliferation, migration and invasion by Wnt/ $\beta$-catenin pathway. Eur Rev Med Pharmacol Sci. 2019;23(18):7855-7862.

25. Liu L, Cao J, Zhao J, Li X, Suo Z, Li H. PDHA1 Gene Knockout In Human Esophageal Squamous Cancer Cells Resulted In Greater Warburg Effect And Aggressive Features In Vitro And In Vivo. Onco Targets Ther. 2019;12:9899-9913.

26. Li S, Huang P, Gan J, Ling X, Du X, Liao Y, Li L, Meng Y, Li Y, Bai Y. Dihydroartemisinin represses esophageal cancer glycolysis by down-regulating pyruvate kinase M2. Eur J Pharmacol. 2019;854:232-239.

27. Xu M, Wang L, Ouyang M, Lin J, Wang L, Zheng X, Miao S, Tang K. Prediction of lymph node metastasis by PET/CT metabolic parameters in patients with esophageal squamous cell carcinoma. Nucl Med Commun. 2019;40(9):933-939.

28. Liu H, Luo J, Luan S, He C, Li Z. Long non-coding RNAs involved in cancer metabolic reprogramming. Cell Mol Life Sci. 2019;76(3):495-504. 
29. Massari F, Ciccarese C, Santoni M, lacovelli R, Mazzucchelli R, Piva F, Scarpelli M, Berardi R, Tortora G, Lopez-Beltran A, Cheng L, Montironi R. Metabolic phenotype of bladder cancer. Cancer Treat Rev. 2016;45:46-57.

30. An J, Zhang Y, He J, Zang Z, Zhou Z, Pei X, Zheng X, Zhang W, Yang H, Li S. Lactate dehydrogenase A promotes the invasion and proliferation of pituitary adenoma. Sci Rep. 2017;7(1):4734.

31. Wang L, Cho KB, Li Y, Tao G, Xie Z, Guo B. Long Noncoding RNA (IncRNA)-Mediated Competing Endogenous RNA Networks Provide Novel Potential Biomarkers and Therapeutic Targets for Colorectal Cancer. Int J Mol Sci. 2019;20(22):5758.

32. An Y, Furber KL, Ji S. Pseudogenes regulate parental gene expression via ceRNA network. J Cell Mol Med. 2017;21(1):185-192.

33. Ghafouri-Fard S, Esmaeili M, Taheri M. Expression of non-coding RNAs in hematological malignancies. Eur J Pharmacol. 2020;875:172976.

34. Ali Syeda Z, Langden SSS, Munkhzul C, Lee M, Song SJ. Regulatory Mechanism of MicroRNA Expression in Cancer. Int J Mol Sci. 2020;21(5):E1723.

\section{Figures}



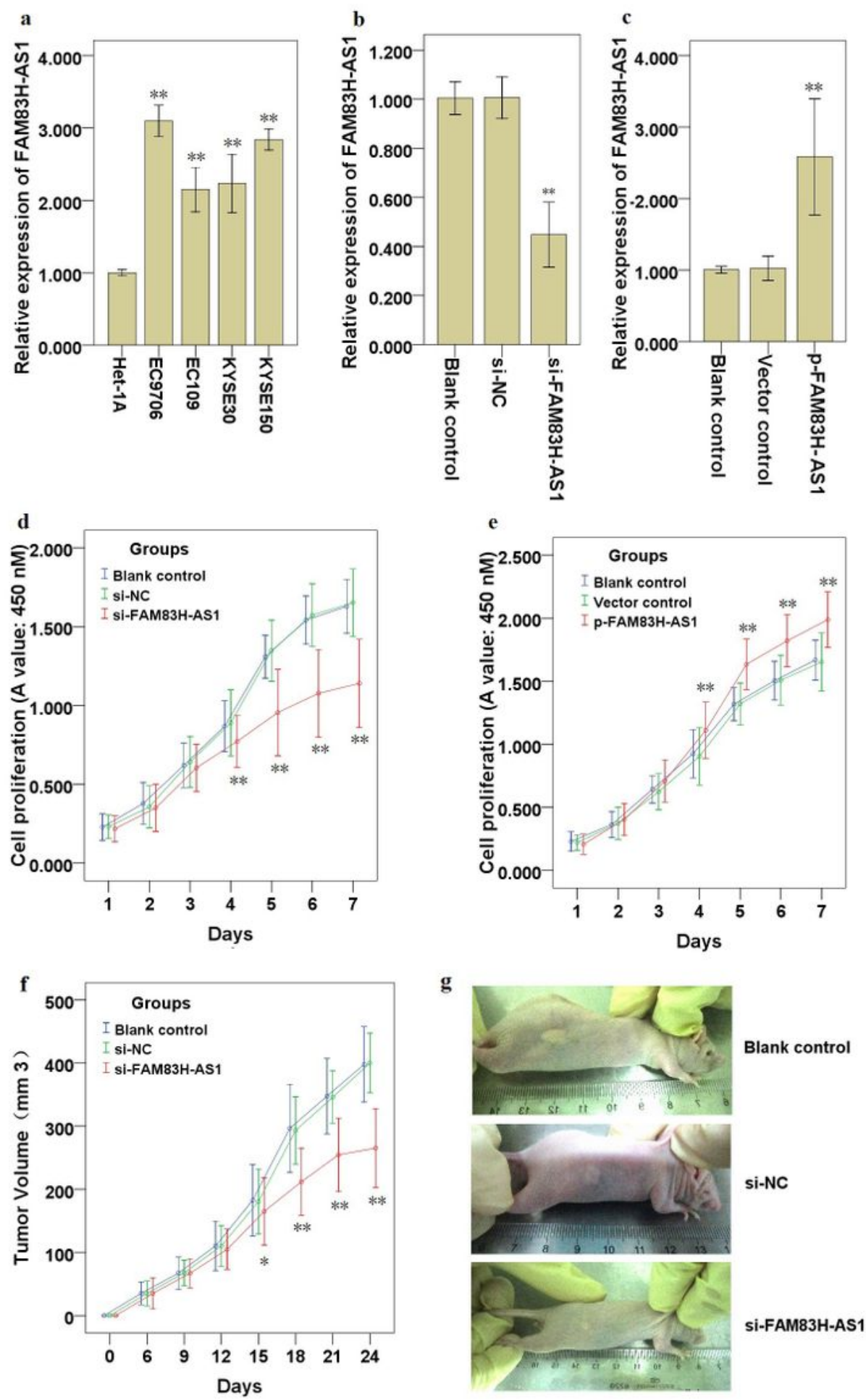

\section{Figure 1}

FAM83H-AS1 modulates the cell proliferation of ESCC cells in vitro and in vivo. a The expression of FAM83H-AS1 in ESCC cell lines and Het-1A cells was examined by qRT-PCR. Gain and loss of functional experiments with the plasmid transfection to silence or enforce the FAM83H-AS1 expression in EC9706 (b) and EC109 (c) cells. CCK-8 assay was performed to detect the cell proliferation in EC9706 (d) and EC109 (e) cells. $\mathrm{f}$ In vivo heterotransplantation mice assay illustrated the tumor volume of EC9706 cell 
neoplasm. g Representative pictures of the nude mice with appropriate controls was shown. ${ }^{*} P<0.05$, and $\star \star P<0.01$ vs control.
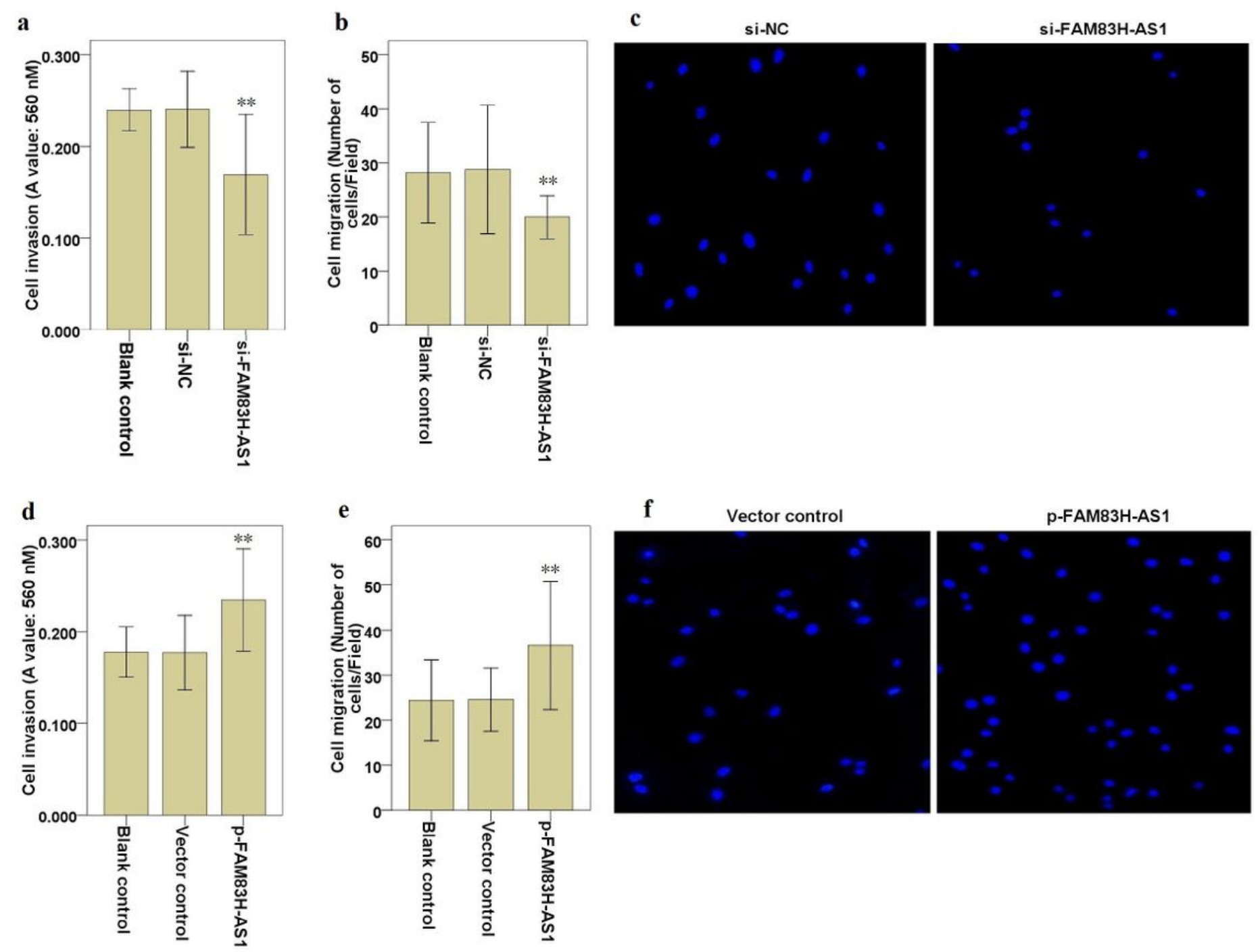

\section{Figure 2}

FAM83H-AS1 modulates cell invasion and migration in ESCC cells. Transwell assay with Matrigel was performed to detect the cell invasion in EC9706 (a) and EC109 (d) cells. Transwell assay without Matrigel was performed to detect the cell migration in EC9706 (b) and EC109 (e) cells. Representative pictures of the cell migration in EC9706 (c) and EC109 (f) was shown. ${ }^{*} \mathrm{P}<0.05$, and $* * P<0.01$ vs control. 

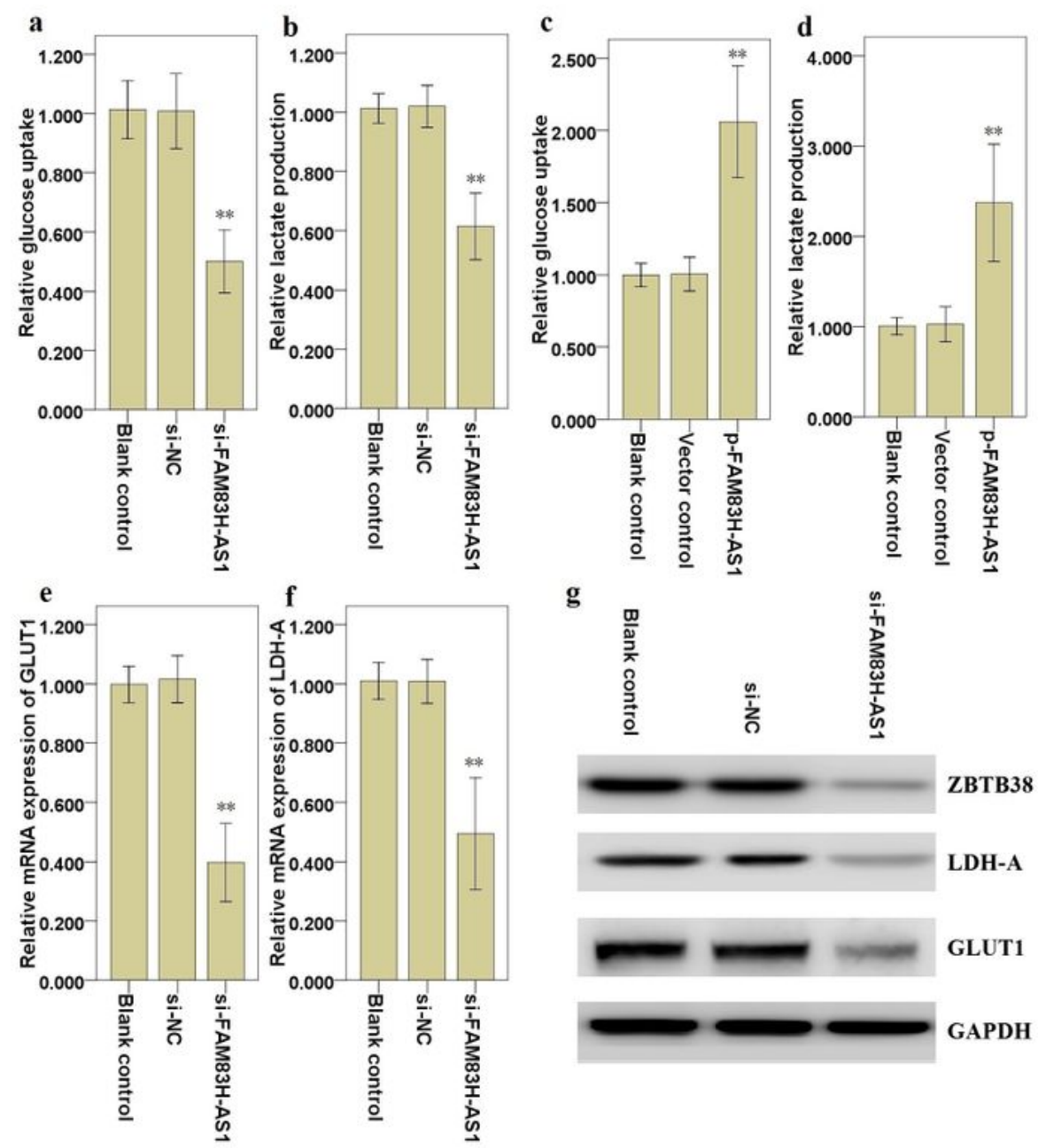

g
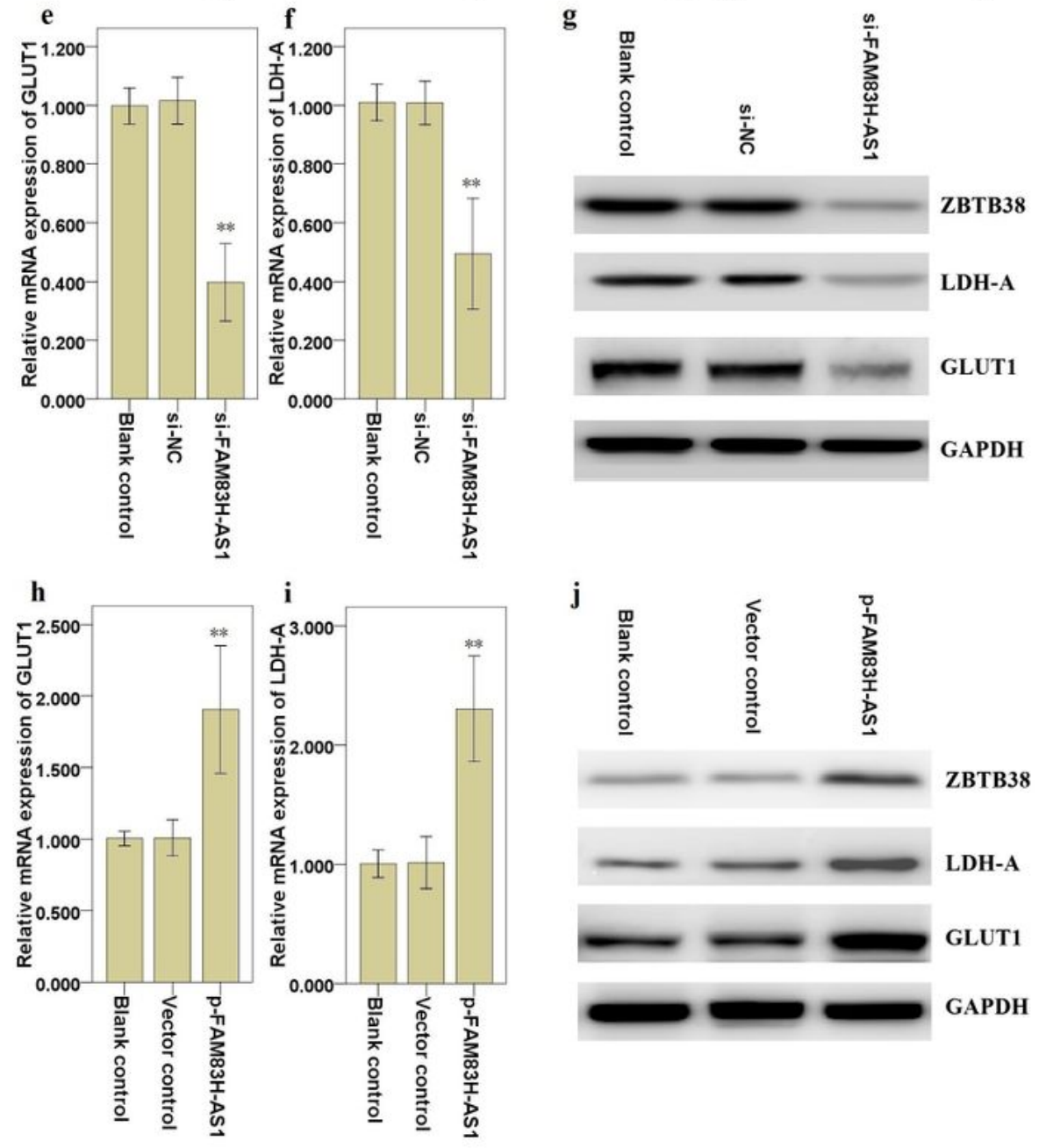

\section{Figure 3}

FAM83H-AS1 affects the aerobic glycolysis in ESCC cells. Effects of FAM83H-AS1 knockdown on the glucose uptake (a) and lactate production (b) in EC9706 cells; Effects of FAM83H-AS1 overexpression on the glucose uptake (c) and lactate production (d) in EC109 cells. qRT-PCR analysis of GLUT1 (e) and LDHA (f) expression following the transfection of EC9706 cells with scrambled siRNA (si-NC) or si-FAM83HAS1; qRT-PCR analysis of GLUT1 (h) and LDH-A (i) expression following the transfection of EC109 cells 
with empty vector (vector control) or pcDNA-FAM83H-AS1 (p-FAM83H-AS1). Western blot analysis of ZBTB38, GLUT1 and LDH-A expression in EC9706 cells transfected with si-NC or si-FAM83H-AS1 (g). EC109 cells transfected with vector control or $\mathrm{p}$-FAM83H-AS1 (j). ${ }^{*} \mathrm{P}<0.05$, and ${ }^{*} \mathrm{P}<0.01$ vs control.

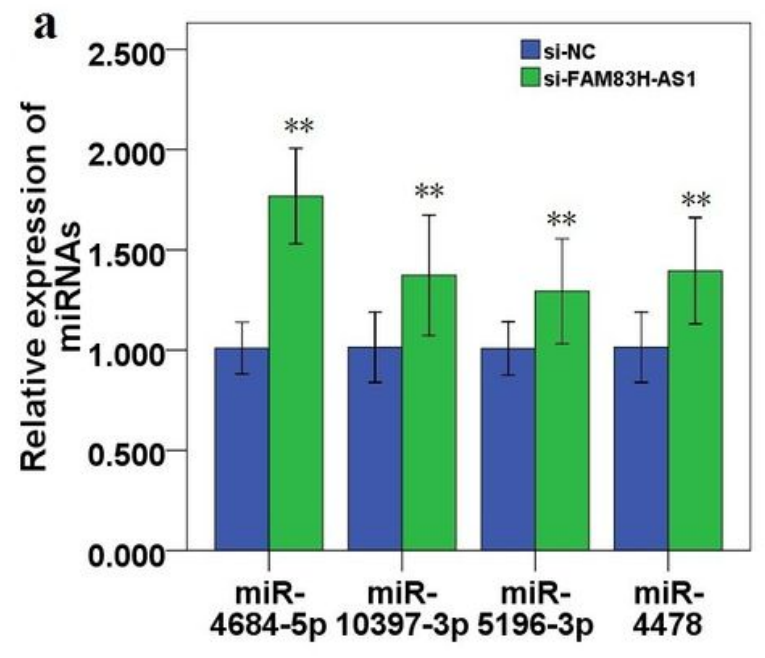

b

FAM83H-AS1 (400-421)-MUT 5'- TCTCTACTTTTTTGTGTTTTT-3'

FAM83H-AS1 (400-421)-WT 5'- AGAGATGATtTtTgtgtTTTT-3'

|||||| $\mid$

miR-4684-5p 3'- TCTCTACTGACTTGCAACATA-5'

c

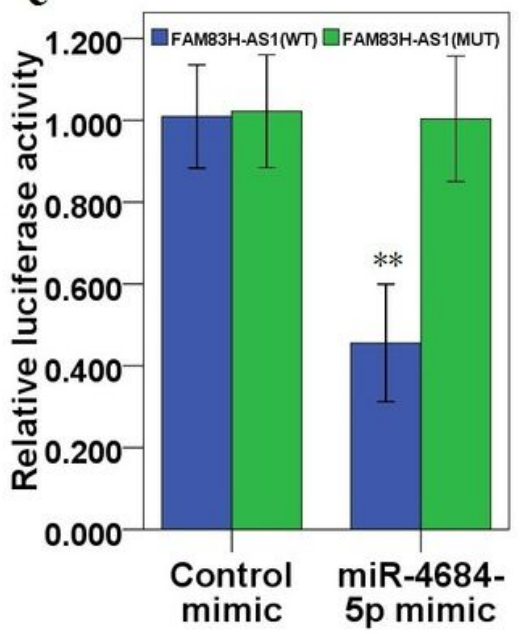

d

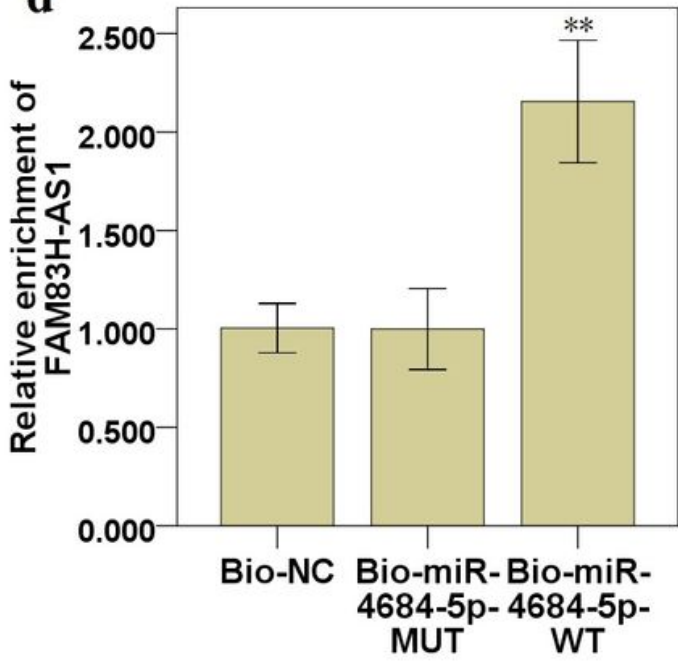

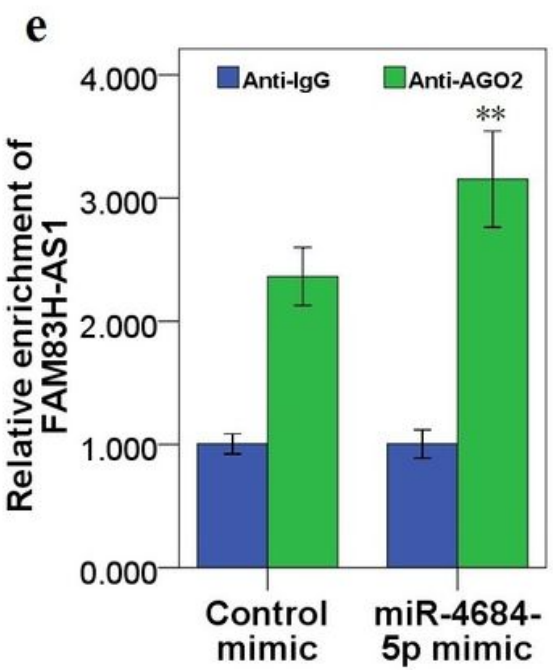

\section{Figure 4}

FAM83H-AS1 bound to miR-4684-5p in ESCC cells. a After transfection of si-FAM83H-AS1, the expressions of miR-4684-5p, miR-10397-3p, miR-5196-3p and miR-4478 were detected via qRT-PCR. $b$ Bioinformatics analysis tools suggested that miR-4684-5p might share the binding sites with FAM83HAS1. c Luciferase reporter assay indicated the molecular level combination of miR-4684-5p and FAM83HAS1. d FAM83H-AS1 was pulled down by biotinylated miR-4684-5p-WT. e AG02-RIP followed by qPCR to detect FAM83H-AS1 enrichment level after miR-4684-5p overexpression via miR-4684-5p mimics. $* P<0.05$, and $* * P<0.01$ vs control. 


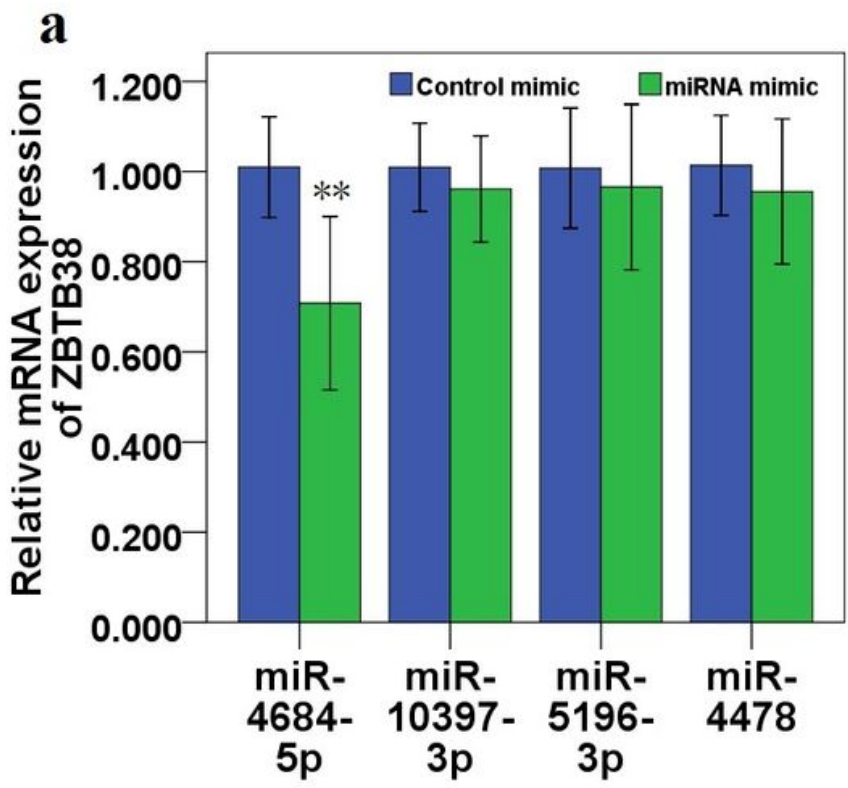

b

ZBTB38 3' UTR 5'-CUGUCCAUAUCCCCUCAUCUCUA-3'
(650-657)-MUT

ZBTB38 3' UTR 5'-CUGUCCAUAUCCCCUGUAGAGAA-3' (650-657)-WT

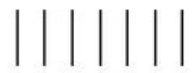

miR-4684-5p 3'-AUACAACGUUCAGUCAUCUCUC-5'
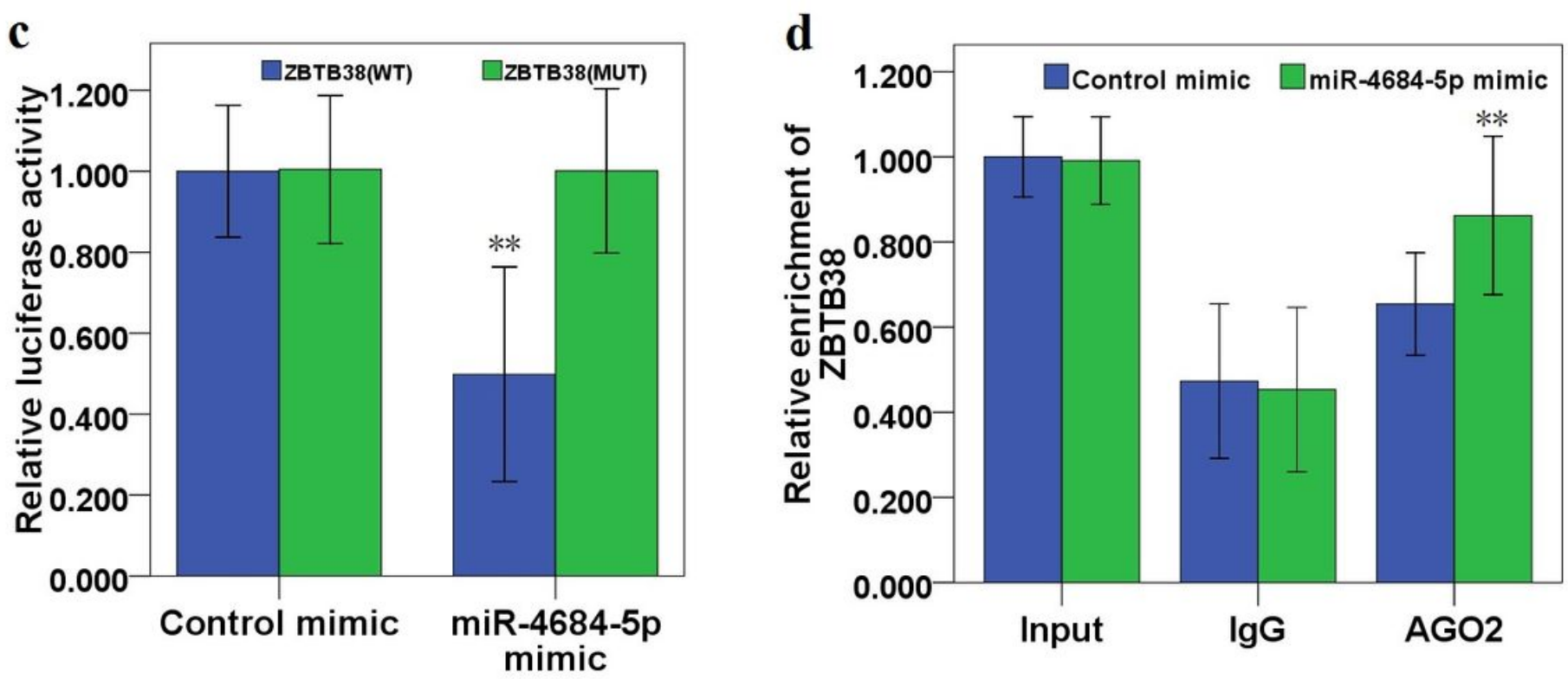

Figure 5

ZBTB38 was a direct target of miR-4684-5p in ESCC cells. a The mRNA expression levels of ZBTB38 were detected via qRT-PCR in EC9706 cells treated by the predicted target miRNA mimics or control mimics. $b$ Bioinformatics analysis tools suggested the associability within miR-4684-5p and ZBTB38. c Luciferase reporter assay indicated the covalent targeting of miR-4684-5p with ZBTB38 mRNA 3'-UTR in EC9706 cells. $d$ RIP assays using antibodies against AGO2 or IgG were performed in cellular lysates from EC9706 cells, and qRT-PCR demonstrated the relative enrichment of ZBTB38 in EC9706 cells transfected with miR-4684-5p or control mimics. ${ }^{*} \mathrm{P}<0.05$, and ${ }^{*} \mathrm{P}<0.01$ vs control. 
a

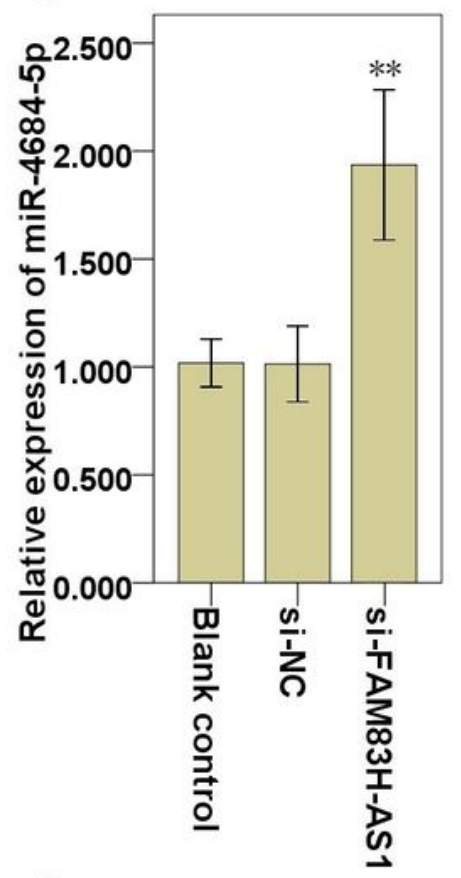

b

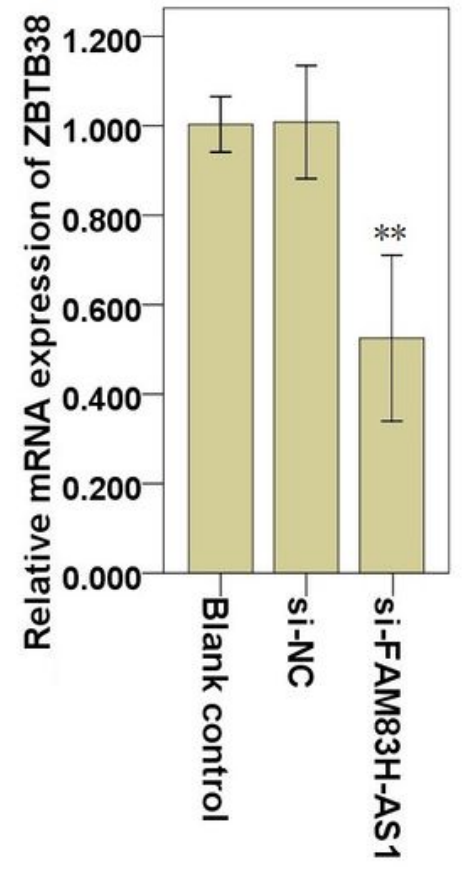

c
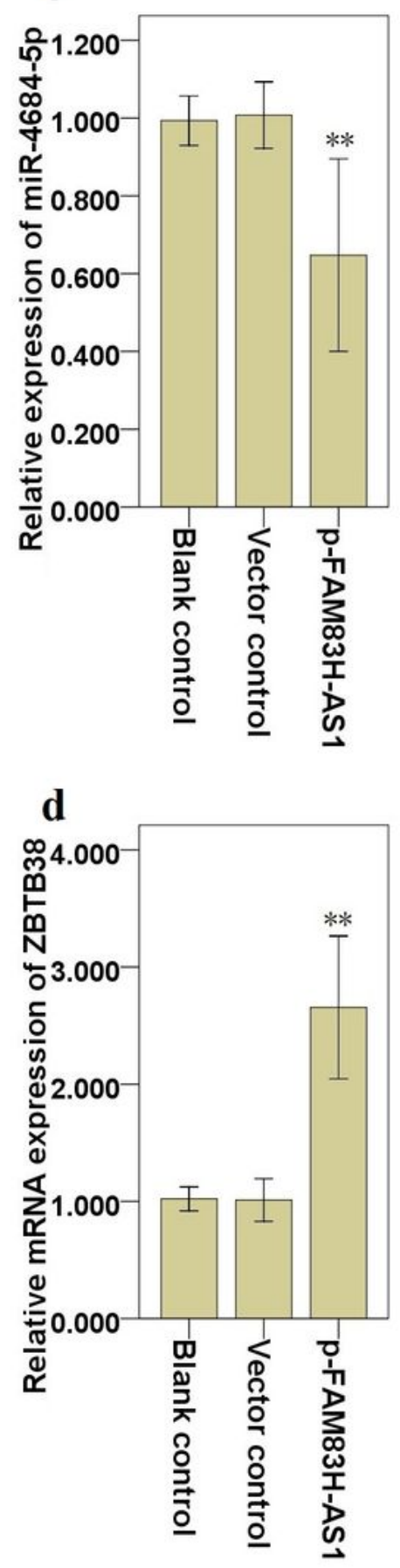
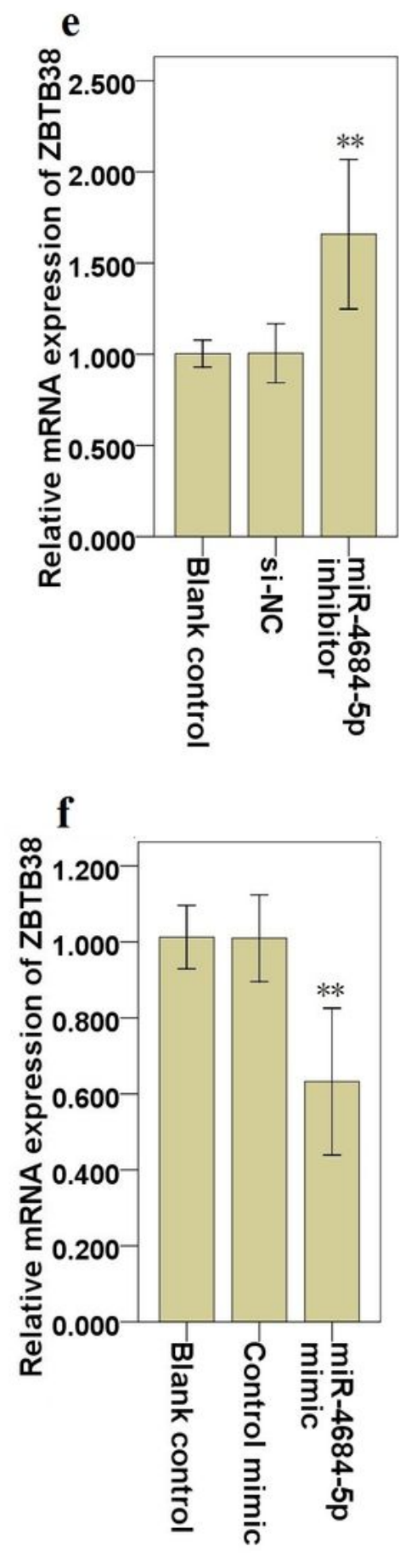

\section{Figure 6}

FAM83H-AS1 modulated miR-4684-5p and ZBTB38 expression, and miR-4684-5p modulated ZBTB38 expression in ESCC cells. qRT-PCR determined the miR-4684-5p expression in EC9706 cells transfected with scrambled siRNA or si-FAM83H-AS1 (a) and EC109 cells transfected with empty vector or p-FAM83HAS1 (c). qRT-PCR determined the ZBTB38 mRNA expression in EC9706 cells transfected with scrambled siRNA or si-FAM83H-AS1 (b) and EC109 cells transfected with empty vector or p-FAM83H-AS1 (d). qRT- 
PCR determined the ZBTB38 mRNA expression in EC109 cells transfected with scrambled siRNA or miR4684-5p inhibitor (e) and EC9706 cells transfected with control mimic or miR-4684-5p mimic (f). ${ }^{*} \mathrm{P}<0.05$, and ${ }^{*} \mathrm{P}<0.01$ vs control. 